\title{
Semi-Automatic Registration of 3D Orthodontics Models from Photographs
}

\author{
Raphaël Destrez $^{* a, c}$, Sylvie Treuillet ${ }^{\mathrm{a}}$, Yves Lucas ${ }^{\mathrm{a},}$ Benjamin Albouy-Kissi ${ }^{\mathrm{b}}$ \\ ${ }^{a}$ Laboratoire PRISME, Polytech'Orléans 12 rue de Blois, 45067 Orléans, France \\ ${ }^{\mathrm{b}}$ Université d'Auvergne, ISIT, BP 10448, 63000 Clermont-Ferrand, France \\ ${ }^{\mathrm{c}}$ XTREMVIZ, 20 rue du 22 Septembre, 92400 Courbevoie
}

\begin{abstract}
In orthodontics, a common practice used to diagnose and plan the treatment is the dental cast. After digitization by a CTscan or a laser scanner, the obtained 3D surface models can feed orthodontics numerical tools for computer-aided diagnosis and treatment planning. One of the pre-processing critical steps is the 3D registration of dental arches to obtain the occlusion of these numerical models. For this task, we propose a vision based method to automatically compute the registration based on photos of patient mouth. From a set of matched singular points between two photos and the dental 3D models, the rigid transformation to apply to the mandible to be in contact with the maxillary may be computed by minimizing the reprojection errors. During a precedent study, we established the feasibility of this visual registration approach with a manual selection of singular points. This paper addresses the issue of automatic point detection. Based on a priori knowledge, histogram thresholding and edge detection are used to extract specific points in $2 \mathrm{D}$ images. Concurrently, curvatures information detects 3D corresponding points. To improve the quality of the final registration, we also introduce a combined optimization of the projection matrix with the 2D/3D point positions. These new developments are evaluated on real data by considering the reprojection errors and the deviation angles after registration in respect to the manual reference occlusion realized by a specialist.
\end{abstract}

Keywords: orthodontics, visual registration, 3D mesh, projection matrix, optimization, point extraction

\section{INTRODUCTION}

In orthodontics, the widespread approach to diagnose and plan the treatment is the dental cast. This manual practice can be replaced by new imaging tools using 3D surface digital models of dental casts. Even if a lot of imaging tools can be used (radiographies, CT-scan or face scan devices [1]) dental casts are often required. 3D numerical models can be obtained from surface laser scans or $100 \mu \mathrm{m}$ resolution CT-scan [2]. Dental casts can be completed by dentition photos [3]. One of the pre-processing critical steps is the registration of 3D dental arches to obtain the occlusion of these numerical models corresponding to the real occlusion of patient. This information can be obtained with a silicone device used during the teeth printing [4], another reference [5] or CT-scan [6] but it requires additional operation for the practitioner and can be expensive. In practice, this occlusion registration step is carried out manually with mouth photos as reference. The result of this process is operator dependent. The main objective of this work is to develop an automatic registration tool to meet the natural occlusion of the 3D arcades models based on patient mouth photographs (Figure 1). With this strategy, we avoid the addition of another step to the current orthodontic practice.

In previous work [7], we proved the feasibility and efficiency of this registration method by using multiple points of views based on a manual selection and matching of singular points.

* raphael.destrez@gmail.com 

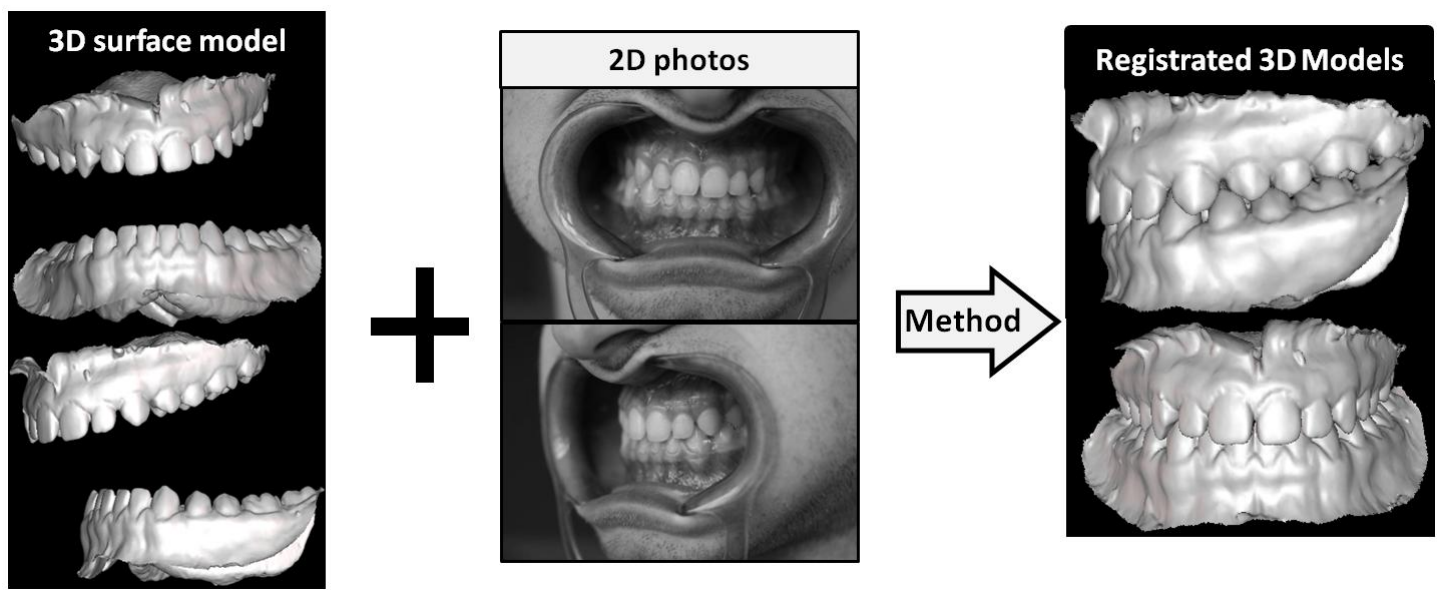

Figure 1.3D model registration by 2D/3D matching between photos and 3D dental arch models.

The aim of this current work is to automatically detect 2D/3D points and test optimization to compute the rigid transformation to apply to place the two arches in natural occlusion. In the following, we present firstly the global strategy used in the process. Secondly, we describe how to extract corresponding points in mouth photos and 3D models using a priori knowledge. Thirdly, we tackle the projection matrix estimation problem before to describe the last step of the registration algorithm. In section 5, we show and discuss some results that illustrate the presented algorithms. Finally, we conclude about the global method and expose several perspectives.

\section{MATERIALS AND REGISTRATION STRATEGY OVERVIEW}

\subsection{Materials}

3D surface models have been obtained from the digitization of patient dental cast by a $100 \mu \mathrm{m}$ resolution CT-scan; model rendering and processing are implemented with VTK [8] and OpenCV libraries. For the point extraction tests, several photos obtained from an uncalibrated camera are used whereas for the other section tests, the real pictures used have been taken with a numerical 8 Mpixels camera (Canon EOS 350D), a $60 \mathrm{~mm}$ lens and an annular flash. This camera has been calibrated. To evaluate the final registration, we rely on the reference pose obtained by the orthodontist expert with the current manual technique.

\subsection{Registration strategy}

The registration consists in the determination of a rigid transformation setting in occlusion the mandible against the maxillary guided by mouth patient photos using several viewpoints. To estimate the rigid transformation, the link between surface model and pictures is a set of correspondences: some points $\boldsymbol{M}_{j}$, selected on 3D surface models may be relied to their projections $\widetilde{\boldsymbol{m}}_{\boldsymbol{i} j}$ in the image $i$, by the projection matrix $\boldsymbol{P}_{i}$ [9]:

$$
\tilde{m}_{i j} \propto \boldsymbol{P}_{i} \boldsymbol{M}_{j}
$$

where $\propto$ means « by a scaling factor ». The $3 \times 4$ projection matrix $\boldsymbol{P}_{i}$ can be decomposed into a product of two matrices:

$$
\boldsymbol{P}_{i}=\boldsymbol{K}\left[\boldsymbol{R}_{i} \mid \boldsymbol{t}_{i}\right]
$$

where $\boldsymbol{t}_{i}$ and $\boldsymbol{R}_{i}$ are respectively the translation and the rotation defining the pose of the camera and $\boldsymbol{K}$ is the camera calibration matrix. 
The registration is realized in two steps: firstly the projection matrix is estimated for each view based on a set of $2 \mathrm{D} / 3 \mathrm{D}$ correspondences selected on the maxillary arch, next the registration of the mandible is realized in respect of maxillary position to obtain the good occlusion according to image. Indeed, the minimized quantity during the optimization process is the sum of the squared reprojection errors:

$$
s e_{i}=\sum_{j=1}^{n_{i}} \sum_{k=1}^{2}\left[m_{i j k}-\widetilde{m}_{i j k}\right]^{2}
$$

where $\mathrm{i}, \mathrm{j}$, and $\mathrm{k}$ are respectively picture, point and coordinate indexes, $m_{i j k}$ refers to the coordinates of matched points measured in the pictures and $\widetilde{m}_{i j k}$ refers to the reprojection of the corresponding 3D point by the matrix $\boldsymbol{P}_{i}$.

In a previous work [7], some preliminary registration results were presented based on a manual selection of $2 \mathrm{D} / 3 \mathrm{D}$ corresponding points. To provide a fully automated process, the critical issue is the detection of some singular corresponding points on the two arches both on the 3D models and on color pictures. Next section deals with this problem. The global registration process is illustrated on the figure 2.

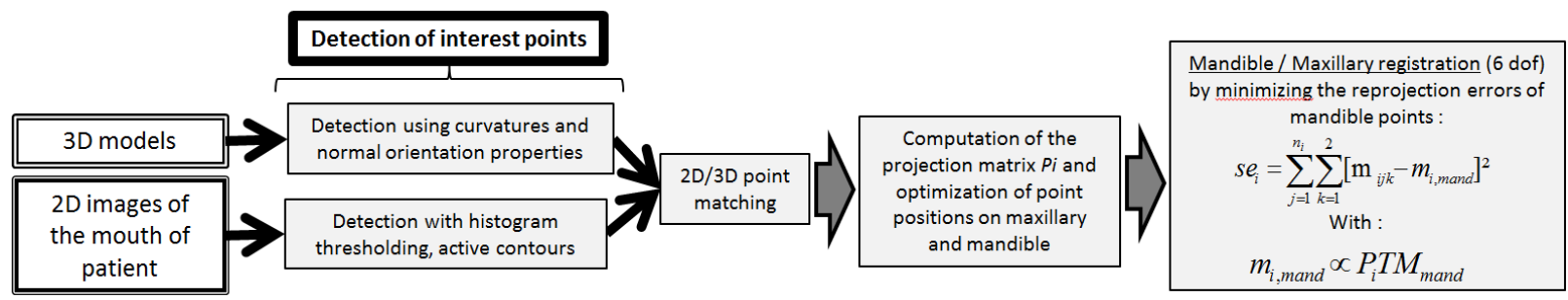

Figure 2. Overview of the registration method

\section{AUTOMATIC DETECTION OF 2D/3D SINGULAR POINTS}

2D/3D singular points have to be simultaneously detected in 3D models and color images. Several interesting points may be extracted along the teeth neck (outline between tooth and gengiva). Three types of singular points are considered on arches: the Interstice points - that separate teeth on mandible or maxillary; the Occlusal points - the middle point of the occlusal front of each tooth and the Middle points - the top of the outline of each tooth neck (figure 3 and 4).

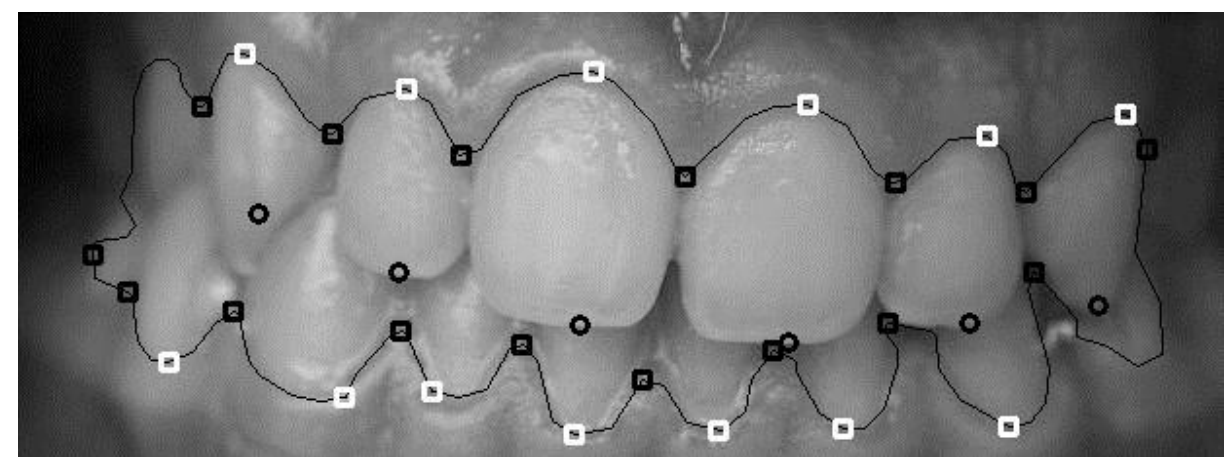

Figure 3. Three types of points are considered for matching: Interstices points (black squares), Occlusal points (black circles) and Middle points (white square) 


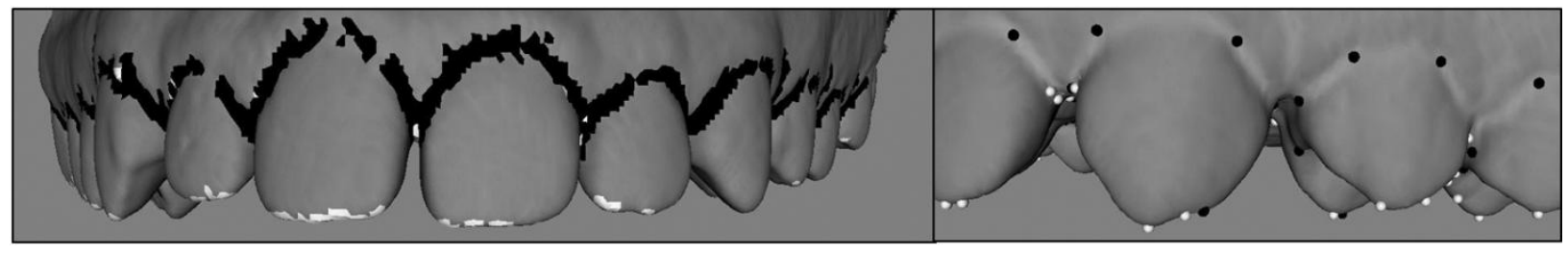

Figure 4. (Left) Extraction of interest areas on the 3D model using curvature information: teeth neck (black) and occlusal area (white); (Right) Extraction of singular point candidates: Interstices points (black) and Occlusal points (white)

The automatic detection of candidate points for matching is not an easy task because data are heterogeneous: on the one hand, 3D models provide only curvatures information, and on the other hand pictures display only color texture. Consequently, we propose two different detection algorithms adapted to each data type.

\subsection{Detection of singular points in color images}

The singular points are detected from a curvature analysis of the teeth neck (outline between tooth and gengiva). The teeth neck is firstly extracted by thresholding on one color channel. The optimal threshold is obtained by splitting the histogram in two parts by applying iterative process from an initial threshold deduced from the histogram shape. Numerous combinations of color channels has been tested with three thresholding methods on a large color image database (40 patients providing each one 3 images): a balanced histogram method $(\mathrm{BH})$, a simple iterative method, sort of one-dimensional case of the K-means algorithm (Auto) and Otsu's method (Otsu) [10]. A pre-processing step of color correction is applied using white patch method by selecting the whitest area in the image. In addition, some postprocessing morphologic transformations are realized to keep finally only the larger central area corresponding to teeth. For comparative study, the resulting outline obtained with each method is compared to the manually labelized ground truth (GT). Table 1 presents the obtained results with the comparative Pratt's criterion ranging between 0 and 1 which represents the best result) [11]. Only relevant portions of the upper part (Up) and the lower part (Down) have been separately considered for evaluation. The best results are obtained with the BH method on hue channel from HSV space. Multi-channel active contour methods like Chan and Vese [12] or narrow band active contours [13] have also been tested on several color spaces including classical or hybrid ones $\left(\mathrm{Ha}^{*} \mathrm{~b}^{*}\right.$, for example). Even if the outline may be improved on a few cases as shown on figure 5 , active contour are not sufficiently robust on our image database.

Table 1. Pratt's evaluation criterion for the three automatic thresholding methods depending on the color channel.

\begin{tabular}{|c|c|c|c|c|c|c|}
\hline & \multicolumn{2}{|c|}{ BH } & \multicolumn{2}{|c|}{ Auto } & \multicolumn{2}{|c|}{ Otsu } \\
\hline Mean \pm SD & Up & Down & Up & Down & Up & Down \\
\hline $\mathbf{H}$ & $0,513 \pm 0,230$ & $\mathbf{0 , 5 3 4} \pm \mathbf{0 , 2 3 7}$ & $0,375 \pm 0,199$ & $0,389 \pm 0,181$ & $0,0957 \pm 0,212$ & $0,091 \pm 0,206$ \\
\hline$a^{*}$ & $0,366 \pm 0,258$ & $0,318 \pm 0,230$ & $0,401 \pm 0,202$ & $0,332 \pm 0,193$ & $0,323 \pm 0,216$ & $0,317 \pm 0,164$ \\
\hline
\end{tabular}
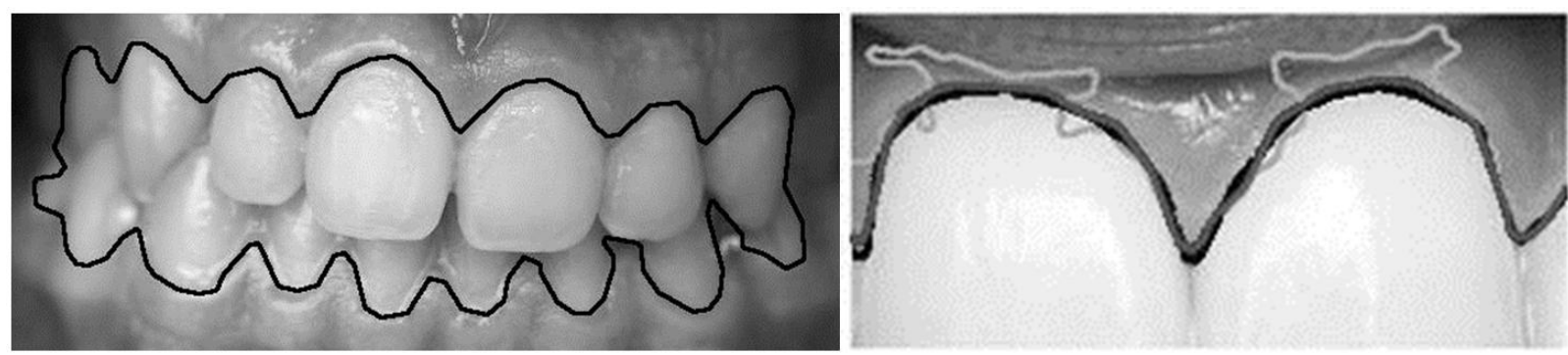

Figure 5. (Left) Detection of the teeth neck outline by thresholding on $\mathrm{H}$ channel by BH method; (Right) Example of an improvement obtained by active contour method Chan \& Vese : reference outline (black), example of an outline obtained by an automatic thresholding (light gray), contour obtained by Chan \& Vese (dark gray). 
Once the teeth neck outlines are extracted, the three types of singular points can be detected. The Interstice and Middle singular points are selected by applying a curvature analysis along the extracted teeth neck outline, considering some $a$ priori knowledge. For example, for Interstice points areas are selected by thresholding the higher curvature. Then, for each area, the point with the ymax or the ymin is extracted respectively for the maxillary and the mandible. Occlusal points are then deducted from the positions of the two previous points on the edge extracted by a difference of Gaussian filters. An example of the three types of singular points detected by this method is presented on Figure 3.

\subsection{Detection of singular points in surface models}

The same three types of singular points are extracted in 3D models by applying different thresholding strategies on various curvatures (principals, Gaussian and mean curvatures). In the area of extreme curvatures extracted around the teeth, other criteria applied for selected the singular points like normal orientation.

For each area of interest, only the point which maximizes or minimizes curvature is selected for the $2 \mathrm{D} / 3 \mathrm{D}$ matching process (see example on Figure 4).

For example, for the extraction of the Interstice points, a combination of a thresholding on the minimum curvatures and the Gaussian curvature are performed to keep only a specific area. After, in this subset, the point maximizing a criterion mixing maximum curvature and triangle normal orientation is chosen to be a candidate for the matching. In the same way, Occlusal point areas are characteristic of a high Gaussian curvature and the Middle points, placed on the teeth neck, can be found for low minimum curvature values (figure 4).

In conclusion, the proposed detection methods permit to automatically extract some interesting candidate points for 2D/3D matching on the two arches, despite the heterogeneous nature of data. Even if the positions of these singular points are not really precise, these positions would be good initialization for the optimization process described in the following section. The corresponding points in images and 3D models are temporally linked by a manual matching for validate the registration, in this work. Automatic matching will be realized in the future by applying teeth segmentation and order constraints on the extracted points.

\section{COMBINED OPTIMIZATION OF THE PROJECTION MATRIX WITH POINT POSITIONS FOR REGISTRATION}

As explained in section 2, the mandible registration is realized from a set of 2D/3D correspondences, by minimizing the reprojection error on several views. Previously, the projection matrix of each view is computed from corresponding pairs selected only on the maxillary. During this calculation, the positions of $2 \mathrm{D}$ and $3 \mathrm{D}$ corresponding points may also be optimized to improve the precision, since the points which are automatically detected may be noisy. But, the number of degrees of freedom (DOF) may be carefully considered as the number of visible singular points in images is very limited. If we considered a calibrated camera, we have to estimate 6 DOF for each camera position ( 3 for translation +3 for rotation). Considering $n$ corresponding 2D/3D points observed in $M$ images, the DOF is $3 n$ for the $3 \mathrm{D}$ positions of points on the models, and $2 n * M$ for the 2D image positions. On the other hand, one correspondence between a 3D point and its $2 \mathrm{D}$ projection in an image provides two equations. So the number of available equations for $M$ images is:

$$
\mathrm{NbEq}=2 * \mathrm{M} * \mathrm{n}
$$

Combined optimization of projection matrix with point positions is realized in four steps (figure 6). Firstly, an initial estimation of the projection matrix $P i$ is computed by using the POSIT algorithm from DeMenthon [14], following by optimization step with Levenberg-Marquardt algorithm [15] [16]. From this initialization, a new combined optimization is done by applying some local constrains to optimize concurrently point positions and the projection matrix Pi. Finally, from the new point positions, the last estimation of $\mathrm{Pi}$ can be done. 


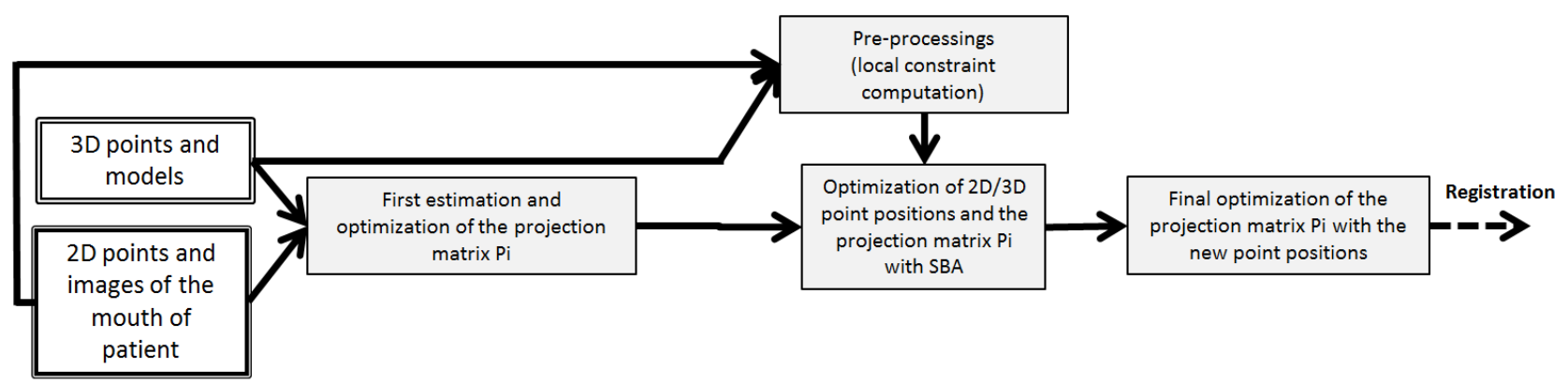

Figure 6. Global optimization of the projection matrix with the point positions.

In the next sub-sections, we describe several approaches for the projection matrix and point position optimization step: firstly, only optimizing the 3D positions on models, secondly only optimizing the 2D positions in images, and finally sequentially optimizing the $2 \mathrm{D}$ positions after the $3 \mathrm{D}$ positions. As the number of parameters to estimate during is very large in respect with the number of visible points, we try to limit the DOF in these different approaches.

\subsection{Combined optimization of projection matrix with 3D positions on models}

To solve this optimization problem by moving only 3D points on the model, the number of equations has to be equal or higher than the number of parameters. From (4), we can deduce the inequality:

$$
2 * \mathrm{M} * \mathrm{n} \geq 6 \mathrm{M}+3 \mathrm{n}
$$

In the case of a pair of images (M=2), the formula (2) entails that a minimum of $n \geq 12$ points is sufficient. Nevertheless, it is recommended to increase $n$ or decrease the number of DOF to improve the result. Moreover, it is necessary to impose some constraints to keep the 3D points on the model mesh during or after the optimization of their coordinates.

For the optimization, the minimized term is the reprojection error between the projection of 3D points by the estimated projection matrix and their correspondents in 2D pictures (equation 3). Three algorithms have been implemented and tested:

A) A classical Sparse Bundle Adjustment (SBA) [17] is used to optimize the M projection matrix Pi and the $n$ point positions [11]. 3D points are moved without constraint to stay on the surface model: points can be move freely according to $\mathrm{X}, \mathrm{Y}$ and $\mathrm{Z}$ components. At the end of the process, the obtained 3D points are projected on the nearest edge of the 3D dental model.

B) The previous process is similarly used except that the 3D point estimated is projected on the $3 \mathrm{D}$ surface model at each iteration step, instead of only at the end of the optimization process.

C) A different surface parameterization of the 3D mesh is used to reduce the number of parameters by describing each 3D point with $\mathrm{U}$ and $\mathrm{V}$ components. This parameterization is carried out in a pre-processing step computing the local constraints. This parameterization step is carried out during the local constraint preprocessing step. This UV mapping is carried out with the ABF algorithm [18] included in the Roadkill software [19]. An advantage of the third method is that this surface parameterization allows the 3D points to freely evolve on the map without the constraint to stay on mesh triangle edges.

The pre-processing step consists in selecting a subset of the 3D mesh by applying local constraints. For each 3D point to be optimized, the K-th neighbors are considered. Then, 3D points can be moved inside this limited area during the optimization (figure 7). Consequently, we decrease the computation time and avoid unlikely estimated 3D point positions. 


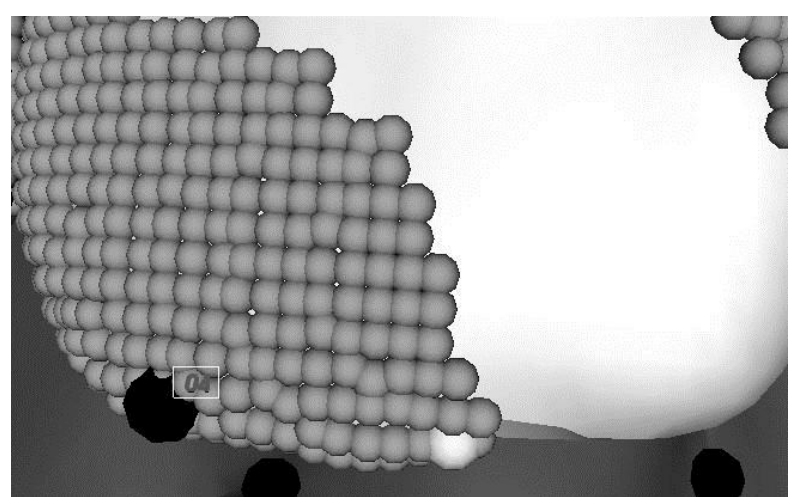

Figure 7. A subset of the mesh defined starting from a point to optimize (black sphere) and including all successive K-th neighbors (gray spheres) with $\mathrm{K}=12$.

\subsection{Combined optimization of projection matrix with $2 \mathrm{D}$ positions in images}

To solve this optimization problem, the number of equations has to be equal or higher than the number of parameters so:

$$
2 * M * n \geq 6 M+2 n * M
$$

Clearly, this inequality is impossible to check so the number of DOF needs to be reduced. To achieve this, the displacement of 2D points is constrained to stay on the outline built for their detection. Consequently, point position is determined by only one curvilinear coordinate and the inequality (6) can be revised in (7), respecting for $n \geq 6$.

$$
2 * \mathrm{M} * \mathrm{n} \geq 6 \mathrm{M}+\mathrm{n} * \mathrm{M}
$$

As in the previous algorithms, the optimization is done by minimizing the reprojection error (equation 3). In this case, we use the Levenberg-Marquardt algorithm (LM) to estimate the projection matrix moving each 2D point along its respective outline. The curve of the discrete outline is interpolated as a continuous curve to allow sub-pixel precision.

\subsection{Combined optimization of projection matrix with 2D/3D point positions}

From (4), (7) and (5) with a UV parameterization of the 3D mesh, the following inequality should be respected to simultaneously optimize $2 \mathrm{D}$ and $3 \mathrm{D}$ point positions:

$$
2 \mathrm{M} * \mathrm{n} \geq(6+\mathrm{n}) * \mathrm{M}+2 \mathrm{n}
$$

To check this condition, we need to carry out the process with 3 images and 18 corresponding points at least. This objective seems difficult to be achieved. So, we propose a sequential optimization process: by mixing the previous described methods, 3D point positions are refined and after 2D positions.

\subsection{Registration of the mandible arch}

The registration of the mandible in respect with the maxillary is realized as described in [7] by estimating a rigid transformation composed of three rotations and three translations. The reference coordinate system of the maxillary is illustrated on figure 8: the origin is the gravity center of the point cloud of the maxillary, the Y axis is facing up and the $\mathrm{Z}$ axis is parallel to the rows of molars. As the reference frame is on the maxillary, 3D points $\boldsymbol{M}_{\text {mand }}$ are projected using the matrix $\boldsymbol{P}_{i}$ estimated from the maxillary points to obtain its projection $\boldsymbol{m}_{i, \text { mand }}$ on the image $i$ :

$$
\boldsymbol{m}_{i, \text { mand }} \propto \boldsymbol{P}_{i} \boldsymbol{T} \boldsymbol{M}_{\text {mand }}
$$


The rigid transformation $\mathrm{T}$ is estimated by minimizing the reprojection errors so that $3 \mathrm{D}$ point projections of the mandible are superimposed with the projections selected in the pictures. The errors between points $\boldsymbol{m}_{i, \text { mand }}$ and their correspondents selected on the picture are minimized to estimate the real occlusion of arches. We consider, simultaneously, two viewpoints for the registration.

Rotation is defined using the Bryant convention from the Euler angles.

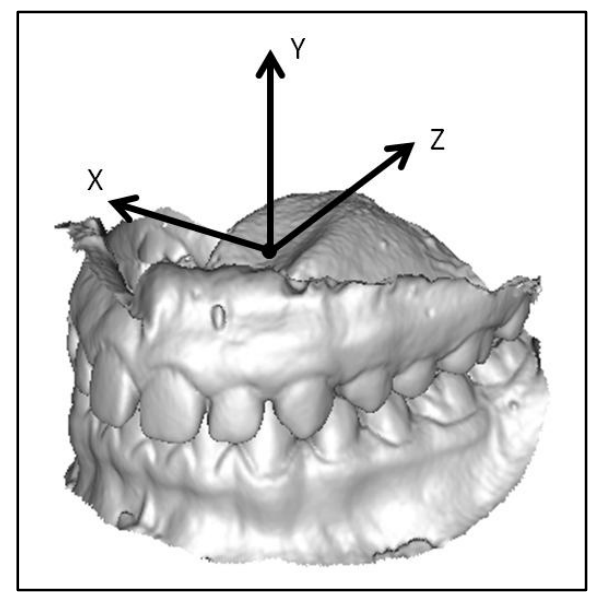

Figure 8. Reference coordinate system of the registration.

\section{EXPERIMENTS AND RESULTS}

\subsection{Experiments}

Our objective is to reach or even improve the precision previously obtained by manual point selection [7], for projection matrix estimation and registration errors. In our experiments, we used similar conditions which are used in previous study [7]: two mouth pictures (a front and a side views) and the corresponding pair of dental arches. To evaluate the registration, the initial pose of the mandible is always the same. This initial pose is defined from the occlusion reference pose by applying on the mandible the following rigid transformation: $(\alpha, \beta, \gamma)=(-15,-6,6)$ for Bryant angles in degrees and $(X, Y, Z)=(-24,-11,-5)$ for translation values in millimeters.

In our experiments, we selected 13 points for the maxillary and 11 points for the mandible. For the 3D point position optimization, the three methods (A, B and C) described in 4.1 have been tested, followed or not by a $2 \mathrm{D}$ position optimization.

For these tests, we fixed $\mathrm{K}=12$ to define the neighborhood where to apply the local constraints to limit the $3 \mathrm{D}$ point displacement on the mesh.

Due to the lack of points $(n=11)$, it is not possible to apply the $(A)$ or $(B)$ methods to optimize the mandible 3D point positions. Consequently, whatever the method used to optimize 3D point positions on maxillary, the method (C) is used for the mandible.

To evaluate the tests, two criteria are observed: the reprojection error which is the minimized quantity in all optimization processes and the 3D registration errors. For the presented evaluation, we used the reprojection mean squared error (MSE), computed for each image (front or side view):

$$
m s e_{i}=\sqrt{\frac{1}{n_{i}} \sum_{j=1}^{n_{i}} \sum_{k=1}^{2}\left[m_{i j k}-\widetilde{m}_{i j k}\right]^{2}}
$$

A little reprojection error signifies generally valuable projection matrix estimation. In [7], we proved that a good estimation of the projection matrix $P i$ is a prerequisite to obtain a good automatic registration. 
The quality of the final registration is evaluated by the deviations on the six parameters of the rigid transformation in respect with the reference occlusion pose. The reference pose is the one obtained by the orthodontist expert with the current technique. The six parameters are the three Bryant angle $(\alpha, \beta, \gamma)$ in degrees and the translation components $\mathrm{X}$, Y and $\mathrm{Z}$ in $\mathrm{mm}$.

To evaluate the efficiency and the feasibility of ours methods, we compare the results obtained with outlines detected by manual or automatic thresholding presented in section 3. The outlines extracted by manual thresholding or automatic thresholding seem to be close, except for few specific areas (figure 9). But globally, the automatic outline fits worse the teeth neck than the manual thresholding extraction. In each case, the selection of the singular points is realized by using the automatic method described in section 2. Consequently, the positions of the 2D points candidates extracted from automatic thresholding are weakly noisy.

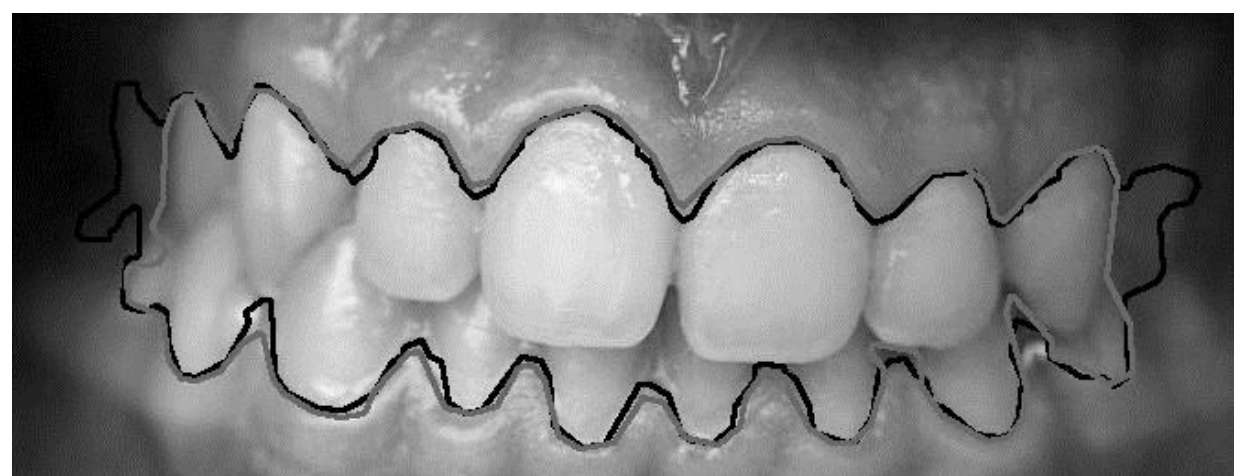

Figure 9. Superimposition of outlines obtained by a manual thresholding (black) and an automatic thresholding (gray)

\subsection{Results}

Tables 2 and 3 are concerned with manual thresholding outlines since the tables 4 and 5 are concerned with automatic thresholding outline. The tables 2 and 4 present the reprojection error (Mean Square Error \pm Standard Deviation in pixels). Moreover, the first line of table 2 contains values for the manual method [7] which we can considered as the reference results and the second line presents the errors before optimization. Registration errors are displayed in table 3. Registration errors obtained in [7] with manual point selection and those obtained without point position optimization process are presented in the two first line of table 3 . The table 4 presents reprojection MSE before and after the optimization by the method which seems to have the best results using the fully automatic point extraction. The table 5 contains registration errors after registration with or without point position optimization.

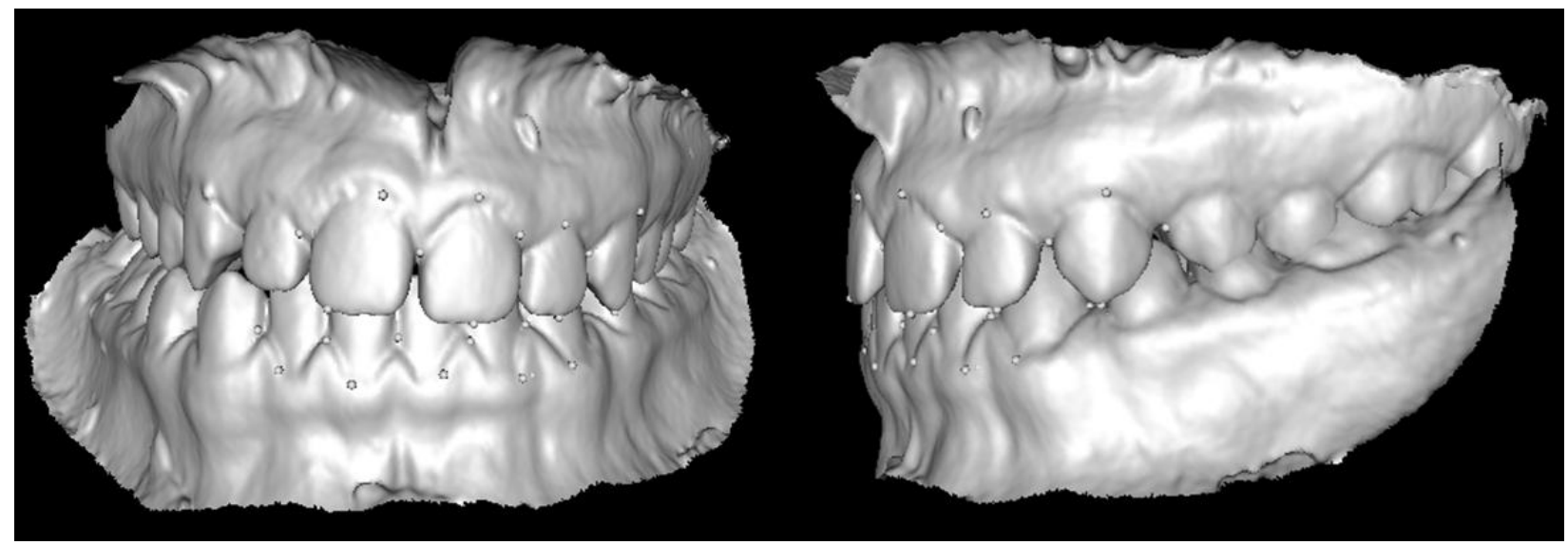

Figure 10. Final registration of mandible for an automatic thresholding outline case with an optimization of 2D point positions step 
Table 2.Reprojection errors before and after optimization depending on the applied method.

\begin{tabular}{|c|c|c|c|c|c|}
\hline & \multicolumn{2}{|c|}{ Front view } & \multicolumn{2}{|c|}{ Side view } \\
\hline & & Maxillary & Mandible & Maxillary & Mandible \\
\hline \multicolumn{2}{|c|}{ Manual method [7] } & $5.24 \pm 2.72$ & $8.38 \pm 2.90$ & $5.44 \pm 2.68$ & $7.70 \pm 3.08$ \\
\hline \multicolumn{2}{|c|}{ Before optimization } & $12.65 \pm 7.52$ & $9.49 \pm 3.30$ & $10.76 \pm 5.98$ & $10.35 \pm 5.12$ \\
\hline \multirow{2}{*}{\multicolumn{2}{|c|}{ Optimization strategy }} & \multicolumn{2}{|c|}{ After optimization } & \multicolumn{2}{|c|}{ After optimization } \\
\hline & & Maxillary & Mandible & Maxillary & Mandible \\
\hline \multirow{3}{*}{$\begin{array}{l}\text { 3D points } \\
\text { optimization }\end{array}$} & A & $7.54 \pm 3.52$ & N/A & $8.64 \pm 3.91$ & N/A \\
\hline & B & $4.77 \pm 3.23$ & N/A & $4.22 \pm 2.31$ & N/A \\
\hline & $\mathrm{C}$ & $4.37 \pm 2.91$ & $2.81 \pm 1.47$ & $4.32 \pm 2.59$ & $2.80 \pm 1.39$ \\
\hline \multicolumn{2}{|c|}{$\begin{array}{c}\text { 2D point } \\
\text { optimization }\end{array}$} & $3.60 \pm 2.03$ & $5.03 \pm 2.73$ & $6.31 \pm 4.55$ & $6.13 \pm 3.46$ \\
\hline \multirow{3}{*}{$\begin{array}{c}\text { Sequential } \\
\text { optimization of } \\
\text { 3D and 2D point } \\
\text { positions }\end{array}$} & A & $4.61 \pm 2.71$ & N/A & $6.90 \pm 4.16$ & N/A \\
\hline & B & $2.32 \pm 1.56$ & N/A & $1.71 \pm 0.69$ & N/A \\
\hline & $\mathrm{C}$ & $1.80 \pm 1.09$ & $1.10 \pm 0.63$ & $1.70 \pm 0.86$ & $2.17 \pm 1.24$ \\
\hline
\end{tabular}

Table 3. 3D errors after registration according to different methods for estimating the projection matrix.

\begin{tabular}{|c|c|c|c|c|c|c|c|c|}
\hline \multirow{2}{*}{\multicolumn{2}{|c|}{ Test configurations }} & \multicolumn{4}{|c|}{$\begin{array}{c}\text { 3D errors relative to the translation }(\mathrm{mm}) \\
\text { after registration }\end{array}$} & \multicolumn{3}{|c|}{$\begin{array}{l}\text { 3D errors relative to the rotation } \\
\text { (degrees) after registration }\end{array}$} \\
\hline & & $\mathrm{X}$ & $\mathrm{Y}$ & $\mathrm{Z}$ & $3 \mathrm{D}$ & $\alpha$ & $\beta$ & $\gamma$ \\
\hline \multicolumn{2}{|c|}{ Manual method [7] } & $\begin{array}{c}0.27 \\
\pm 0.21 \\
\end{array}$ & $\begin{array}{r}0.56 \\
\pm 0.22 \\
\end{array}$ & $\begin{array}{c}0.14 \\
\pm 0.16 \\
\end{array}$ & $\begin{array}{l}0.68 \\
\pm 0.2\end{array}$ & $\begin{aligned} & 1.26 \\
& \pm 0.86 \\
&\end{aligned}$ & $\begin{array}{l}0.95 \\
\pm 0.74 \\
\end{array}$ & $\begin{array}{r}0.27 \\
\pm 0.20 \\
\end{array}$ \\
\hline \multicolumn{2}{|c|}{$\begin{array}{c}\text { Without point position } \\
\text { optimization } \\
\end{array}$} & 0.42 & 1.21 & 1.09 & 1.68 & 5.69 & 2.45 & 2.18 \\
\hline \multirow{3}{*}{$\begin{array}{l}\text { 3D points } \\
\text { optimization }\end{array}$} & $\overline{\mathrm{A}}$ & 1.17 & 1.97 & 0.09 & 2,29 & 5,88 & 1.66 & 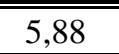 \\
\hline & $\mathrm{B}$ & 0.73 & 0.71 & 1.07 & 1,48 & 3,65 & 2.17 & 1,59 \\
\hline & $\mathrm{C}$ & 0.37 & 1.22 & 0.86 & 1,53 & 4,37 & 1.65 & 2,05 \\
\hline \multicolumn{2}{|l|}{$\begin{array}{c}\text { 2D point } \\
\text { optimization }\end{array}$} & 0.21 & 0.04 & 0.65 & 0,69 & 1,17 & 1.01 & 2,71 \\
\hline \multirow{3}{*}{$\begin{array}{c}\text { Sequential } \\
\text { optimization of } \\
\text { 3D and 2D point } \\
\text { positions }\end{array}$} & $\mathrm{A}$ & 1.99 & 0.53 & 0.46 & 2,11 & 2,55 & 3.36 & 7,73 \\
\hline & $\mathrm{B}$ & 0.59 & 0.25 & 0.77 & 1,00 & 1,36 & 2.68 & 2,33 \\
\hline & $\mathrm{C}$ & 0.20 & 0.17 & 0.76 & 0,81 & 1,99 & 3.3 & 3.2 \\
\hline
\end{tabular}

Table 4. Reprojection error before and after optimization for the fully automatic extraction point case.

\begin{tabular}{|c||c|c||c|c|}
\hline \multicolumn{1}{|c||}{} & \multicolumn{2}{c||}{ Front view } & \multicolumn{2}{c|}{ Side view } \\
\cline { 2 - 3 } & Maxillary & Mandible & Maxillary & Mandible \\
\cline { 2 - 3 } $\begin{array}{c}\text { Before } \\
\text { optimization }\end{array}$ & $12.15 \pm 7.40$ & $9.62 \pm 5.05$ & $13.48 \pm 6.50$ & $11.97 \pm 5.51$ \\
\hline \multicolumn{1}{|c||}{} & \multicolumn{2}{|c||}{ After optimization } & \multicolumn{2}{|c|}{ After optimization } \\
\cline { 2 - 3 } & Maxillary & Mandible & Maxillary & Mandible \\
\hline 2D & $5.09 \pm 2.92$ & $5.07 \pm 2.93$ & $\begin{array}{c}8.57 \pm 5.83 \\
(20.18)\end{array}$ & $8.06 \pm 4.74$ \\
\hline
\end{tabular}


Table 5. 3D errors after registration for the fully automatic extraction point case

\begin{tabular}{|c||c|c|c|c|c|c|c|}
\hline \multicolumn{1}{|c||}{} & \multicolumn{3}{|c|}{$\begin{array}{c}\text { 3D errors relative to the translation }(\mathrm{mm}) \\
\text { after registration }\end{array}$} & \multicolumn{3}{c|}{$\begin{array}{c}\text { 3D errors relative to the } \\
\text { rotation (degrees) after } \\
\text { registration }\end{array}$} \\
\cline { 2 - 8 } & $\mathrm{X}$ & $\mathrm{Y}$ & $\mathrm{Z}$ & $3 \mathrm{D}$ & $\alpha$ & $\beta$ & $\gamma$ \\
\hline $\begin{array}{c}\text { Without point } \\
\text { position } \\
\text { optimization }\end{array}$ & 0.51 & 2.32 & 1.98 & 3.09 & 9.88 & 1.87 & 3.11 \\
\hline 2D & 0.60 & 0.04 & 0.56 & 0.82 & 2.93 & 0.002 & 4.34 \\
\hline
\end{tabular}

\subsection{Discussion}

The first part of the discussion is concerned with the validation of optimization point position and projection matrix estimation step with a teeth neck outline obtained by manual thresholding (tables 2 and 3). Comparing to the errors before optimization, all optimization methods decrease the reprojection errors. Moreover, except the method (A) without 2D position optimization, all strategies obtain better results than the reference manual method. Except for the maxillary on the image 2, the method (C) seems to have the lowest errors and (A) the most important if there is no 2D position optimization after the optimization and the registration. For the method with 2D position optimization only, reprojection errors range from (A) and (B) results. If we optimize 3D and after 2D positions, we can make the observations than in the case without $2 \mathrm{D}$ optimization but generally the results are best and reprojection errors are lower than without $2 \mathrm{D}$ optimization.

The study of 3D registration errors (tables 4 and 5) is a better way to evaluate the real efficiency of this matrix estimation and to evaluate its impact on the complete process. Concerning the 3D point optimization, it's hard to conclude about the best method between (B) and (C), with or without 2D optimization. These two methods improve the registration comparing to the values before optimization. The worst results of the method (A) can be explained by that the positions of 3D points are constrained to be on the 3D mesh only at the end of the optimization process. Then, the projection matrix is computed without considering the 3D mesh, during the optimization of 3D points.

The method that optimizes only the 2D positions seems to obtain the best results. Optimize one parameter by point in image is easier than to move 3D points on a mesh. Except for the Bryant angle $\gamma$, registration errors are equivalent with errors obtained for the reference manual method. Note that this method has not the lowest reprojection. Indeed, reprojection error is more a convergence indicator during the optimization than a quality indicator.

To test the fully automatic point extraction, we applied only the $2 \mathrm{D}$ optimization method. The results are presented in table 4 and 5. We can observe a significant decrease of the reprojection and registration errors after point position optimization in images. The final reprojection errors are similar than those obtained in the reference case of manual point selection. Concerning the registration errors (table 5), the translation deviations are equivalent to the manual reference but the angle deviations are globally higher especially for the Bryant's angle $\gamma$. In spite of this comment, we can observe that the registration obtained (figure 10) is close to a good occlusion, even if there are few collisions.

\section{CONCLUSION}

The proposed vision based approach proves to be promising to solve the orthodontic registration problem using classical mouth photos and 3D models available in orthodontics. Fully automatic point extraction techniques have been carried out for both color images and 3D surfaces models. A 2D/3D matching of some singular points permits to estimate the projection matrix to achieve a registration close to the real occlusion. As point positions computed by automatic extraction are noisy, the proposed optimization algorithms improve the quality of the projection matrix estimation and the registration. But the sparse property of our point extraction strategy is a limiting factor for an accurate registration, even if we decrease the number of parameters. Moreover, to estimate all parameters in the $2 \mathrm{D}$ position optimization algorithm, points are constrained to move along the found outlines and edge. Consequently, the initial extraction of 2D points and their displacement is very dependent from the outlines and edges. 
In the future, our objective is to implement an automatic matching of the 2D/3D points, based on order constraint and iterative estimation for example. Finding a denser extraction method for $2 \mathrm{D}$ and $3 \mathrm{D}$ points could permit to move $2 \mathrm{D}$ point more freely and not along outlines and edges but only constrain by them. Registration results may also be improved by optimizing $2 \mathrm{D}$ and $3 \mathrm{D}$ points concurrently during optimization step than the sequential one currently implemented. Another important issue for the quality of the registration is to consider the collisions between the two dental models during registration process.

\section{REFERENCES}

[1] http://www.ortho-concept3d.com/.

[2] Ettorre, G. et al., "Standards for digital photography in cranio-maxillo-facial surgery - Part I: Basic views and guidelines", Journal of Cranio-Maxillofacial Surgery, 34(2), 65-73 (2006).

[3] Rangel, F. A. \& al., "Integration of digital dental casts in 3-dimensional facial photographs ", American Journal of Orthodontics and Dentofacial Orthopedics, 134(6), 820-826(2008).

[4] Alfano, S. G. and Leupold, R. J., "Using the neutral zone to obtain maxillomandibular relationship records for complete denture patients", The Journal of Prosthetic Dentistry, 85(6), 621-623(2001).

[5] Sohmura T., et al, "High-resolution 3-D shape integration of dentition and face measured by new laser scanner", IEEE transactions on medical imaging, 23(5), 633-638(2004) .

[6] Swennen, G. R. J., et al., "A cone-beam CT based technique to augment the 3D virtual skull model with a detailed dental surface", International Journal of Oral and Maxillofacial Surgery, 38(1), 48-57(2009).

[7] Destrez, R., Albouy-Kissi, B., Treuillet, S., Lucas, Y., Marchadier, A., "Comparison of Visual Registration Approaches of 3D Models for Orthodontics", Proc. ACIVS'11, 647-657 (2011).

[8] http://www.vtk.org/.

[9] Hartley, R. and Zisserman, A.," Multiple View Geometry in Computer Vision", second, 2004, Cambridge University Press, ISBN: 0521540518

[10] Otsu, N., "A threshold selection method from gray-level histograms", IEEE Transactions on Systems, Man, and Cybernetics, 9(1), 62-66 (1979).

[11] Pratt, W. K., Faugeras, O. D. and Gagalowicz, A., " Visual Discrimination of Stochastic Texture Fields", IEEE Transactions on Systems, Man and Cybernetics, 8(11),796 -804(1978).

[12] Chan, T. F. , Sandberg, B. Y. and Vese, L. A. "Active Contours without Edges for Vector-Valued Images", Journal of Visual Communication and Image Representation, 11, 130-141(2000).

[13] Mille, J., "Narrow band region-based active contours and surfaces for 2D and 3D segmentation", Computer Vision Image Understanding, 113( 9), 946-965(2009).

[14] DeMenthon, D.F., Davis, L.S., "Model-based object pose in 25 lines of code", International Journal of Computer Vision, 15, 123--141 (1995).

[15] Lourakis, M. I. A., "levmar: Levenberg-Marquardt nonlinear least squares algorithms in $\{\mathrm{C}\} /\{\mathrm{C}\}++$ ", <http://www.ics.forth.gr/ lourakis/levmar/+> (July 2004).http:/www.ics.forth.gr/ lourakis/levmar/

[16] Marquardt, D., "An Algorithm for Least-Squares Estimation of Nonlinear Parameters", SIAM Journal on Applied Mathematics, 11(2), 431-441(1963).

[17] Lourakis, M. I. A. and Argyros, A. A., "SBA: a software package for generic sparse bundle adjustment", ACM Transactions on Mathematical Software, 36(1), 1-30(2009).

[18] Sheffer, A., de Sturler, E., "Parameterization of Faceted Surfaces for Meshing Using Angle Based Flattening", Engineering with Computers", 17(3), 326-337 (2001).

[19] http://www.pullin-shapes.co.uk/page8.htm 